\title{
11. Appendices
}

\section{Appendix 1. Description of selected congenital conditions}

\begin{tabular}{|c|c|}
\hline \multicolumn{2}{|c|}{ The following include descriptions of some of the congenital conditions included in this report: } \\
\hline Anencephaly & Absence of the cranial vault, with the brain tissue completely missing or markedly reduced. \\
\hline Spina bifida & Incomplete closure of the bony encasement of the spinal cord, through which the spinal cord may protrude. \\
\hline Encephalocele & Protrusion of brain through a congenital opening in the skull. \\
\hline Hydrocephalus & Dilatation of the cerebral ventricles accompanied by an accumulation of cerebral fluid within the skull. \\
\hline Buphthalmos & Enlargement and distension of the fibrous coats of the eye. \\
\hline Hypospadias & The opening of the urethra lies on the underside of the penis or on the perineum. \\
\hline Epispadias & $\begin{array}{l}\text { Absence of the upper wall of the urethra. The opening of the urethra lies on the dorsum of the penis in males, } \\
\text { and anterior to or onto the clitoris in females. }\end{array}$ \\
\hline Chordee & Downward bowing of the penis. \\
\hline Talipes equinovarus & A deformity of the foot in which the heel is elevated and turned outward. \\
\hline Polydactyly & Presence of additional fingers or toes on hands or feet. \\
\hline Syndactyly & Attachment of adjacent fingers or toes on hands or feet. \\
\hline Craniosynostosis & Premature closure of the sutures of the skull. \\
\hline Exomphalos & Herniation of the abdominal contents into the umbilical cord. \\
\hline Gastroschisis & A defect in the abdominal wall not involving the umbilicus and through which the abdominal contents herniate. \\
\hline Cystic hygroma & A sac, cyst or bursa distended with fluid. \\
\hline
\end{tabular}

\section{Appendix 2. Congenital conditions exclusion list}

\begin{tabular}{|c|c|c|}
\hline \multicolumn{3}{|c|}{$\begin{array}{l}\text { The following is a general list of minor conditions and non-structural disorders that are excluded from the NSW Register } \\
\text { of Congenital Conditions: }\end{array}$} \\
\hline Abnormal palmar creases & Haemophilia & Muscular dystrophies \& myopathies \\
\hline Accessory nipples & Heart murmurs (functional) & Oesophageal reflux \\
\hline $\begin{array}{l}\text { Balanced chromosomal translocation } \\
\text { (unless occurring with structural anomalies) }\end{array}$ & $\begin{array}{l}\text { Hernia (epigastric, hiatus, inguinal, umbilical) } \\
\text { Hydrocele (testis) }\end{array}$ & $\begin{array}{l}\text { Patent ductus arteriosus } \\
\text { (less than } 37 \text { weeks gestation) }\end{array}$ \\
\hline Birthmarks (single, $<4 \mathrm{cms}$ diameter) & Hypoplastic lung (less than 37 weeks gestation) & Pilonidal sinus \\
\hline Bronchopulmonary dysplasia & Imperforate hymen & Sacral dimples \\
\hline $\begin{array}{l}\text { Cerebral palsy } \\
\text { Clicky hips }\end{array}$ & $\begin{array}{l}\text { Inborn errors of metabolism other } \\
\text { than phenylketonuria, galactosemia }\end{array}$ & $\begin{array}{l}\text { Single umbilical artery } \\
\text { (unless occurring with structural anomalies) }\end{array}$ \\
\hline Congenital infections & and congenital hypothyroidism. & Skin tag \\
\hline (unless occurring with structural anomalies) & Intrauterine growth retardation & Strabismus \\
\hline Congenital neoplasms/tumours & Low birth weight & Talipes (exception: those requiring surgery) \\
\hline (exception: cystic hygroma) & Meconium ileus & Tongue tie \\
\hline Developmental disability & Minor ear anomalies & Undescended testes \\
\hline Deviated nasal septum & Minor finger/hand anomalies & (exception: those requiring surgery) \\
\hline Fetal alcohol syndrome & Minor toe/foot anomalies & Webbing of 2 nd and $3 r d$ toes \\
\hline $\begin{array}{l}\text { Glucose-6-phosphate dehydrogenase } \\
\text { (G6PD) deficiency }\end{array}$ & & Wide sutures \\
\hline
\end{tabular}




\section{Appendix 3. Maternal countries of birth and country of birth groups}

\begin{tabular}{|c|c|c|c|}
\hline English speaking & Eastern Europe, Russia, Central & Egypt & Southern Asia \\
\hline Australia & Asian and Baltic States & Libya & Afghanistan \\
\hline Christmas Island & Bulgaria & Mauritania & Bangladesh \\
\hline Cocos (Keeling) Islands & Czechoslovakia & Morocco & Bhutan \\
\hline Norfolk Island & Hungary & Sudan & India \\
\hline New Zealand & Poland & Tunisia & Maldives \\
\hline United Kingdom & Romania & Cameroon & Nepal \\
\hline Channel Islands & Armenia & Central African Republic & Pakistan Sri Lanka \\
\hline Isle of Man & Azerbaijan & Congo & \\
\hline Ireland & Belarus (formerly Byelorussia) & Cote d'Ivoire & Southern Europe \\
\hline Bermuda & Estonia & Gambia & Albania \\
\hline Canada & Georgia & Ghana & Andorra \\
\hline United States of America & Kazakhstan & Guinea-Bissau & Cyprus \\
\hline South Africa & Latvia & Liberia & Gibraltar \\
\hline & Lithuania & Mali & Greece \\
\hline Central and South America & Moldova (formerly Moldavia) & Nigeria & Italy \\
\hline Bahrain & Russian Federation & Senegal & Malta \\
\hline Argentina & Ukraine & Sierra Leone & Portugal \\
\hline Bolivia & Uzbekistan & Zaire & Spain \\
\hline Brazil & Kazakhstan & Angola & Bosnia-Herzegovina \\
\hline Chile & Kyrgystan & Botswana & Croatia \\
\hline Colombia & & Djibouti & Macedonia \\
\hline Ecuador & Melanesia, Micronesia & Ethiopia & Slovenia \\
\hline Falkland Islands & and Polynesia & Kenya & Serbia and Montenegro \\
\hline French Guiana & New Caledonia & Malawi & Former Yugoslavia \\
\hline Guyana & Papua New Guinea & Mauritius & (not otherwise defined) \\
\hline Paraguay & Solomon Islands & Mozambique & \\
\hline Peru & Vanuatu & $\begin{array}{l}\text { Namibia } \\
\text { Reunion }\end{array}$ & Western and Northern Europe \\
\hline Surinam & Guam & $\begin{array}{l}\text { Reunion } \\
\text { Rwanda }\end{array}$ & Austria \\
\hline Uruguay & Kiribati & $\begin{array}{l}\text { Rwanda } \\
\text { Seychelles }\end{array}$ & Belgium \\
\hline Venezuela & Nauru & Somalia & France \\
\hline Belize & $\begin{array}{l}\text { Cook Islands } \\
\text { Fiji }\end{array}$ & Swaziland & Germany (United) \\
\hline Costa Rica & $\begin{array}{l}\text { Fiji } \\
\text { French Polynesia }\end{array}$ & Tanzania & Luxembourg \\
\hline El Salvador & $\begin{array}{l}\text { French Polynesia } \\
\text { (including Tahiti) }\end{array}$ & Uganda & Netherlands \\
\hline Guatamala & Niue & Zambia & Switzerland \\
\hline $\begin{array}{l}\text { Honduras } \\
\text { Mexico }\end{array}$ & American Samoa & Zimbabwe & Denmark \\
\hline Nicaragua & Western Samoa & Eritrea & Faeroe Islands \\
\hline Panama & Tokelau & Ethiopia & Finland \\
\hline Antigua and Barbuda & Tonga & & Iceland \\
\hline Bahamas & Tuvalu & North East Asia & Norway \\
\hline Barbados & Wallis and Fortuna & China (excluding Taiwan) & Sweden \\
\hline Cayman Islands & & Hong Kong & \\
\hline Cuba & Middle East and Africa & Japan & \\
\hline Grenada & Bahrain & North Korea & \\
\hline Guadeloupe & Gaza Strip & South Korea & \\
\hline Jamaica & Iran & Macau & \\
\hline Netherlands Antilles & Iraq & Mongolia & \\
\hline Puerto Rico & Israel & Taiwan & \\
\hline St Kitts-Nevis & Jordan & & \\
\hline St Lucia & Kuwait & South East Asia & \\
\hline St Vincent and the Grenadines & Lebanon & Brunei & \\
\hline Trinidad and Tobago & Qatar & Cambodia & \\
\hline \multirow[t]{8}{*}{ Turks and Caicos Islands } & Saudi Arabia & Indonesia & \\
\hline & Syria & Laos & \\
\hline & Turkey & Malaysia & \\
\hline & United Arab Emirates & Burma (Myanmar) & \\
\hline & West Bank & Philippines & \\
\hline & Yemen & Singapore & \\
\hline & Algeria & Thailand & \\
\hline & & Vietnam & \\
\hline
\end{tabular}


Appendix 4. NSW Midwives Data Collection Form

PLEASE PRESS FIRMLY WHEN COMPLETING THIS FORM

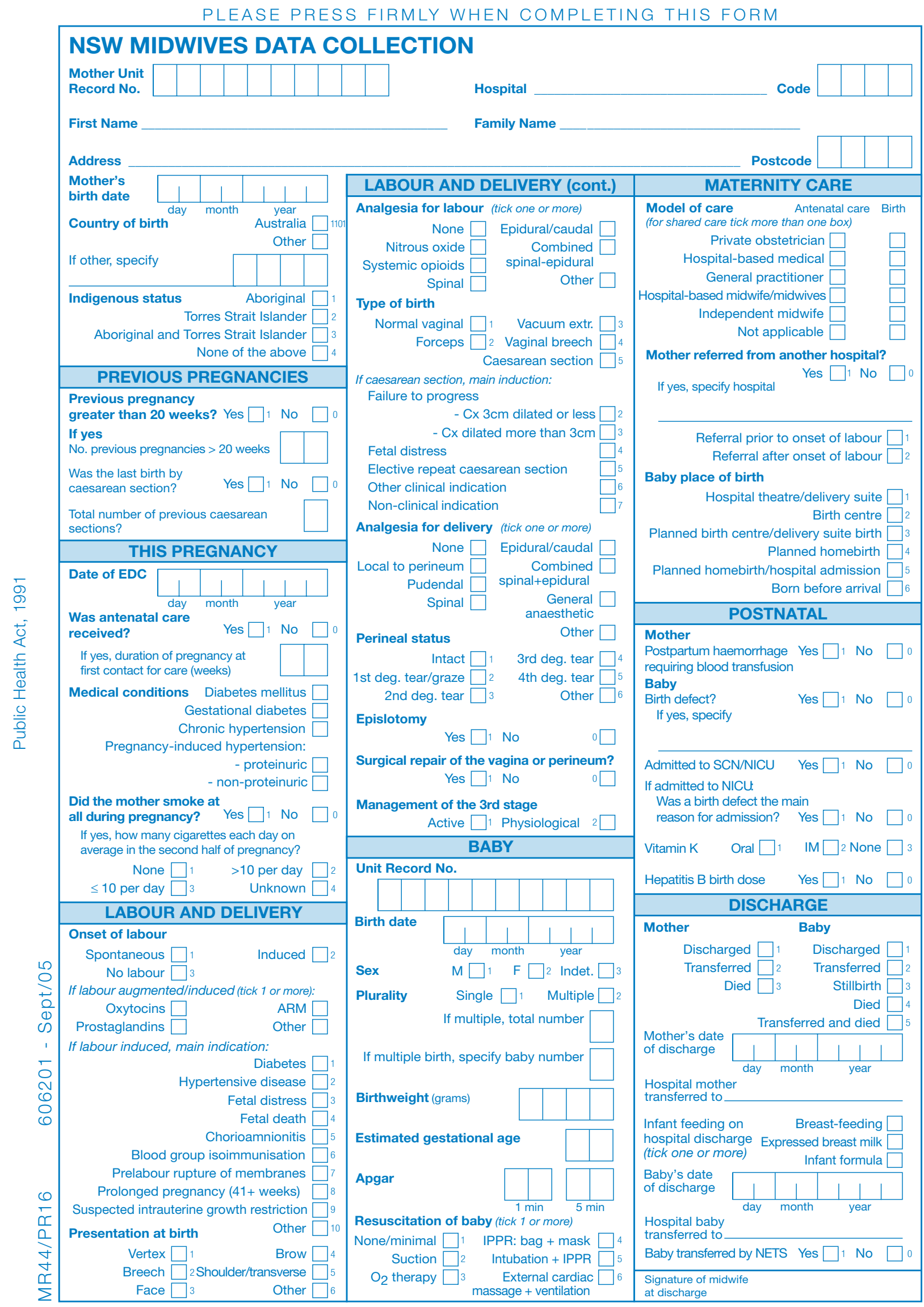

Health Department Copy Please compete and forward to: NSW Midwives Data Collection

Performance Analysis and Reporting Branch, Level 5, NSW Department of Health Locked Bag 961, North Sydney, NSW 2059 